\title{
Art of operative techniques: treatment options in arch penetrating aortic ulcer
}

\author{
Chiara Lomazzi ${ }^{1}$, Viviana Grassi ${ }^{1}$, Maurizio Domanin ${ }^{1,2}$, Carlo De Vincentiis ${ }^{3}$, Gabriele Piffaretti ${ }^{4}$, \\ Santi Trimarchi ${ }^{1,2}$
}

${ }^{1}$ Vascular Surgery, Fondazione IRCCS Cà Granda Ospedale Maggiore Policlinico, Milan, Italy; ${ }^{2}$ Department of Clinical and Community Sciences, University of Milan, Milan, Italy; ${ }^{3}$ Cardiac Surgery Department, IRCCS Policlinico San Donato, San Donato Milanese, Italy; ${ }^{4}$ Vascular Surgery, Department of Medicine and Surgery, University of Insubria School of Medicine, Varese, Italy

Correspondence to: Santi Trimarchi, MD, PhD. Fondazione IRCCS Cà Granda Ospedale Maggiore Policlinico, University of Milano, Via Francesco Sforza 28 20122, Milano, Italy. Email: santi.trimarchi@unimi.it.

\begin{abstract}
Penetrating aortic ulcer (PAU) of the arch has a focal extent which often represents an adequate anatomic target for thoracic endovascular aortic repair (TEVAR). However, the anatomic constraints represented by the supra-aortic vessels pose either clinical or technical challenges that increase when the PAU develops proximally in the arch. Currently, different types of endografts are commercially available and have been used to treat aortic arch lesions. These include branched/fenestrated endografts for a total endovascular approach, and standard devices that can be used in combination with open/hybrid surgical operations, with the aim to exploit the minimally invasive nature of TEVAR by extending the proximal landing zone when necessary. We describe several current techniques adopted in such settings.
\end{abstract}

Keywords: Penetrating aortic ulcer (PAU); hybrid aortic arch repair; thoracic endovascular aortic repair (TEVAR); surgical options

Submitted May 03, 2019. Accepted for publication Jul 22, 2019.

doi: $10.21037 /$ acs.2019.07.06

View this article at: http://dx.doi.org/10.21037/acs.2019.07.06

\section{Introduction}

Penetrating aortic ulcer (PAU) is a focal lesion of the arterial intima and media that generally develops due to a disruption of an atherosclerotic plaque (1). The natural history of PAU is not completely clear, being potentially associated with intramural hematoma (IMH) and aortic dissection or leading to aortic wall rupture (2). Typically, PAUs develop as isolated lesions and are mainly located in the descending thoracic aorta (3), with only $7 \%$ of PAUs in the aortic arch (4). Up to $38 \%$ of PAUs have abrupt onset with rupture-those presenting with pain have been associated with morphologic progression and complication (5). For these reasons, the recent European Society for Vascular Surgery guidelines on the management of thoracic aortic disease recommend the treatment of all complicated PAUs (class IIa) (6). For asymptomatic lesions, surgical or endovascular repair should be considered in the presence of potential predictors such as an ulcer that initially measures $>20 \mathrm{~mm}$ in diameter and $>10 \mathrm{~mm}$ in depth, since these cases were significantly associated with disease progression (7). PAUs are typically detected in older patients compared to those affected by IMH or dissection. For this reason, they present more frequently with comorbidities, often making them unfit for open surgical repair (8). Open surgery has been associated with high morbidity and mortality rates (9-38\%) (9). In contrast, the solitary nature and focal extent of these lesions makes them adequate anatomic targets for thoracic endovascular aortic repair (TEVAR). Although several series have reported favorable short and mid-term outcomes $(3,6-8)$, PAUs developed in the arch are the most challenging to treat endovascularly. The anatomic constraints presented by the supra-aortic vessels pose either clinical or technical challenges that increase with more proximal arch PAU. Currently, different types of endografts are commercially 


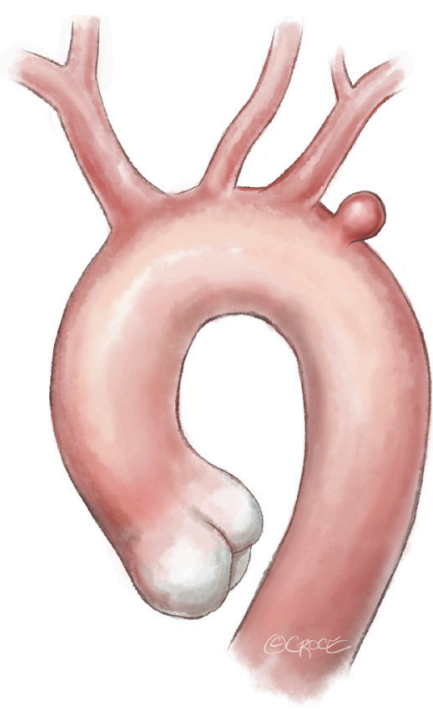

Figure 1 Penetrating aortic ulcer (PAU) of the left hemiarch which requires proximal TEVAR deployment in Ishimaru zone 2. TEVAR, thoracic endovascular aortic repair.

available and have been used to treat aortic arch lesions (10-12). Aside from the most recent total endovascular approach with branched/fenestrated endografts, standard devices have been used in combination with open surgical maneuvers in the so-called "hybrid" operations, with the aim of exploiting the minimally invasive nature of TEVAR by extending the proximal landing zone in those cases unsuitable for standard endovascular management.

\section{Operative techniques}

\section{TEVAR options for treating distal aortic arch PAU (landing zone 2)}

\section{Hybrid options for left subclavian artery (LSA) and left vertebral artery (LVA) revascularization}

When a PAU is located in the proximity of the LSA origin and requires overstenting for secure sealing at the proximal landing zone ("zone 2") (Figure 1), surgical revascularization of the LSA and therefore of the LVA may be performed using two different surgical approaches. The most frequently used technique is the carotid-subclavian bypass with a supra-clavicular surgical access (Figure $2 A$ ); this type of bypass, generally performed with a prosthetic graft, has been increasingly used following the wide adoption of "hybrid" operations in the distal aortic arch. Mainly indicated for elective cases, it may be performed in a two- stage fashion when emergency operations require the intentional overstenting of the LSA. According to the most recent guidelines $(6,13)$, carotid-subclavian bypass graft is recommended for LSA revascularization in most circumstances: long $(>20 \mathrm{~cm})$ descending thoracic aortic coverage, especially in the presence of a previous infrarenal aortic repair for preventing spinal cord ischemia, left coronary artery bypass with the internal mammary artery, absent/hypoplastic right vertebral artery, and left upper limb vascular access for hemodialysis. LSA coverage without revascularization may be performed in emergent settings or when the LVA is non-dominant. Other "hybrid" techniques for LSA revascularization could be considered during TEVAR such as carotid-subclavian transposition or right-to-left axillary bypass graft.

\section{Total endovascular options for LSA and LVA revascularization}

Although different techniques and approaches have been adopted for LSA and LVA revascularization, few studies report robust data with the use of total endovascular solutions. Being predominantly used in urgent settings, the parallel graft techniques either in the chimney or periscope configuration have gained popularity (Figure 2B). Developed as bailout approaches in very demanding circumstances they can offer versatility and adaptability using standard off-theshelf devices (generally less technically demanding). Major concerns are related to the safety of the procedure, with a reported stroke rate up to $7.8 \%$, as well as the absence of long-term data. Given consequences such as strokes, migration, breakage and "gutter-related" endoleaks, which are reported in up to $21 \%$ of the cases, careful assessment is required to determine the safety and durability of the aortic arch reconstruction (14). The most elegant and anatomical total endovascular approach for a "zone 2" lesion is represented by the use of single-arm branched endograft. Currently still not on the market, these devices produced by Gore (W.L. Gore ${ }^{\circledR}$ and ass.; Flagstaff, AZ, USA) and Medtronic ${ }^{\circledR}$ (Medtronic Inc.; Minneapolis, MN, USA) are under evaluation in two different trials to assess their feasibility and results. A further total endovascular solution is represented by the custom-made scallop endograft (Terumo Aortic) (Figure 2C), whose main constraint could be the variability of subclavian artery anatomy that can limit its use in urgent settings since the manufacturer requires three weeks' notice to customize the device (12). A last alternative for LSA preservation is represented by the "in situ" endovascular fenestration using laser-based catheters. 


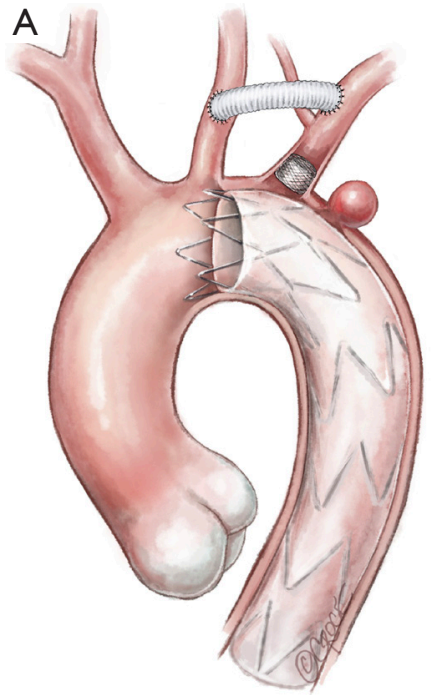

D

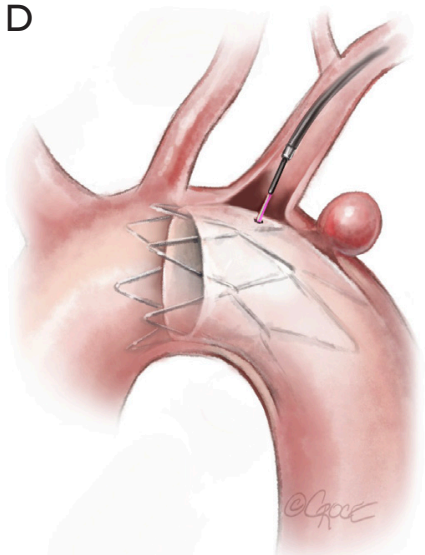

B

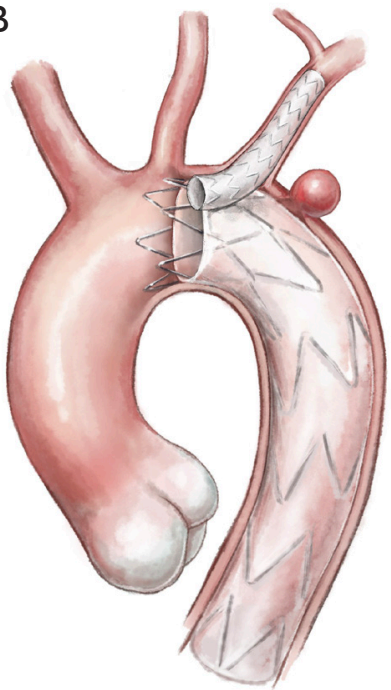

$E$

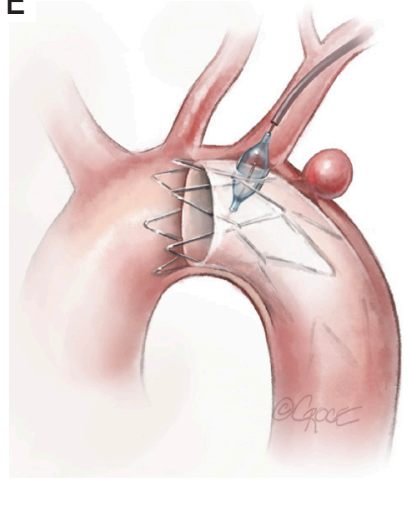

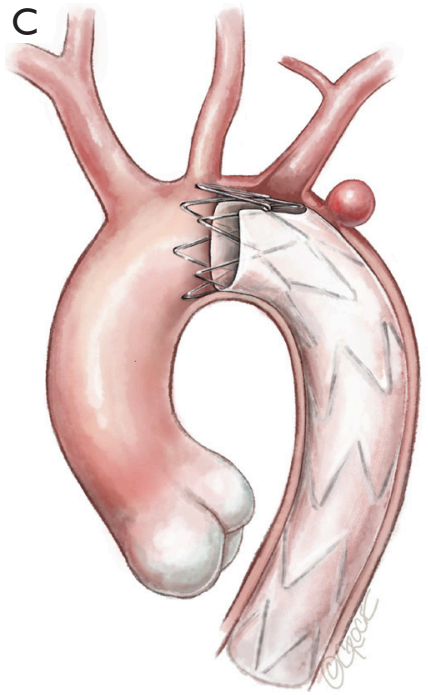

F

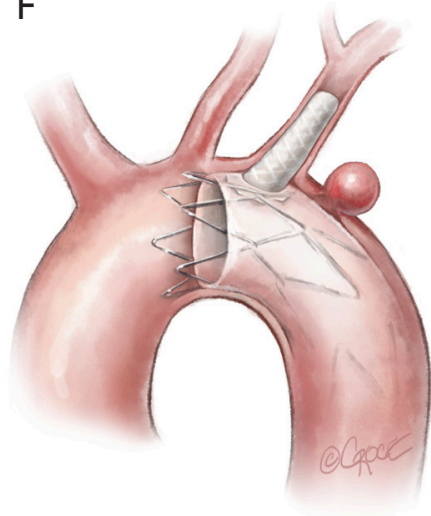

Figure 2 Hybrid and total endovascular techniques for treating PAU which require TEVAR in landing zone 2. (A) Endograft deployment in Ishimaru zone 2 with surgical carotid-subclavian bypass graft and retrograde plug embolization of the LSA. A vascular plug is positioned proximally to the origin of LVA. (B) LSA preservation using the parallel grafts technique. (C) Scallop device for LSA preservation. TEVAR in zone 2 with in situ fenestration for preserving LSA. This technique includes the use of (D) laser catheter for fenestration (E), balloon dilatation and (F) stent positioning. PAU, penetrating aortic ulcer; TEVAR, thoracic endovascular aortic repair; LSA, left subclavian artery; LVA, left vertebral artery.

This procedure is performed through a retrograde upper limb arterial access; once the fenestration catheter is gently advanced to contact the endograft, a hole in the fabric is created, dilated with an angioplasty balloon catheter, and lastly, reinforced and stabilized with a stent (Figure 2D,E,F).

\section{Sizing and preparation}

The patient is placed in the supine position, eventually with mild right-side rotation of the left shoulder. Carotid- subclavian bypass graft is performed under general anesthesia, heparinization and neurologic monitoring (transcranial-Doppler, EEG). Generally, surgical cutdown is performed at the groin to expose the common femoral artery since patients with PAUs frequently have severely calcified, small diameter iliac and femoral vessels that may be prone to complications such as disruption, thrombosis, dissection and false aneurysm. Thus, careful evaluation of the most appropriate and safest access for endograft insertion is mandatory. Occasionally a retroperitoneal exposure of iliac arteries to perform an iliac conduit for 


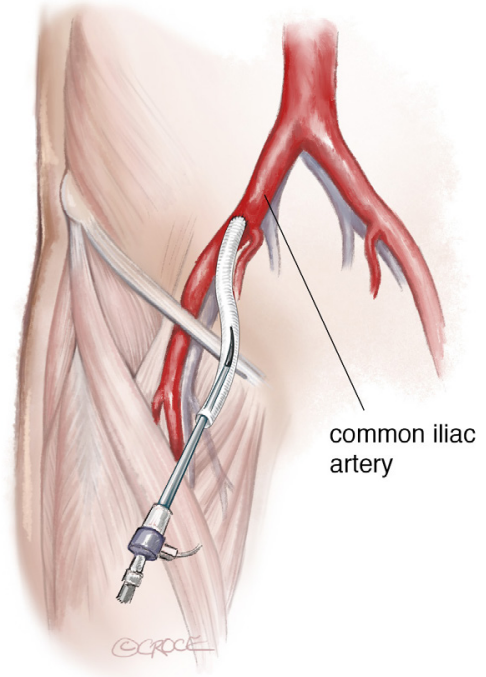

Figure 3 Access for endograft delivery: prosthetic conduit using the iliac artery as an alternative when percutaneous approach is not possible for small and hostile femoral arteries.

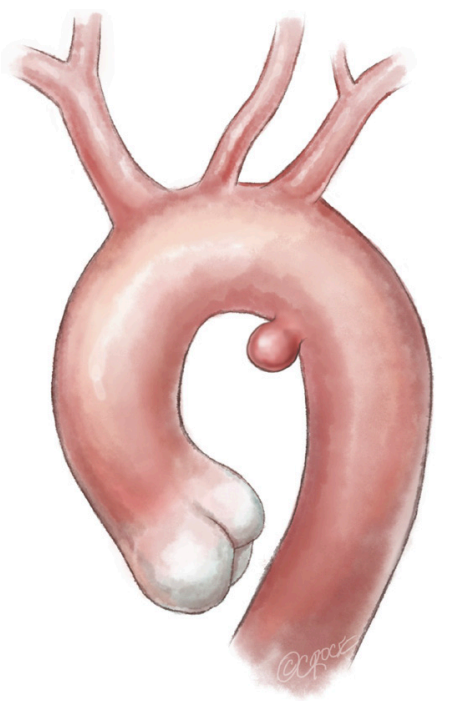

Figure 4 Penetrating aortic ulcer (PAU) of the inner curve of the aortic arch which requires proximal TEVAR deployment in Ishimaru zone 1. TEVAR, thoracic endovascular aortic repair.

endograft delivery is used (Figure 3); in PAU literature, this has been required in up to $46 \%$ of the cases $(6,15)$. To avoid surgical cut-down of the femoral artery in the most favorable cases a percutaneous approach can be planned.

A supra-clavicular longitudinal incision is made to expose both the LSA and the left common carotid artery.
Surgical exposure should be preferentially performed lateral to the neck in order to identify and preserve the brachial plexus and not medially to avoid damage to the thoracic duct, which may lead to chylothorax. Sequential clamping (subclavian first) is used to perform the bypass graft, which should be as short as possible to avoid kinking and tunneled under the internal jugular vein. Repair via left arm percutaneous access or surgical cut-down is used when deploying a scalloped device or parallel graft.

\section{Operation: hybrid procedure for left hemiarch repair}

The endograft is introduced and deployed in "zone 2" (Ishimaru classification)-between the origin of the left common carotid artery and the origin of the LSA. In order to avoid a retrograde type 2 endoleak caused by reversal of flow in the LVA, the LSA is embolized at its origin with an endovascular plug.

\section{Operation: totally endovascular left hemiarch repair}

The endograft is introduced with a femoral percutaneous or surgical cut-down exposure and access from the left arm for a parallel graft to maintain perfusion of LSA. In the parallel graft technique, the LSA chimney stent is planned to be placed (inside the arch) at least $10 \mathrm{~mm}$ proximal to the upper fabric edge of the aortic endograft. The two devices are ballooned using a "kissing balloon technique" as a final procedural step to optimize expansion and mutual adaptation. The additional use of rapid ventricular pacing, which can be used during the repair of all zones of the aortic arch, may play a significant role in reducing endograft misplacement as well as the triggering of stent-graft induced new intimal damages.

TEVAR options for treating left hemiarch PAU (landing zone 1)

\section{Hybrid options for cerebral revascularization}

With the widespread use of "hybrid" operations, TEVAR has challenged conventional surgery in the transverse aortic arch (Figure 4).

Once used only for obstructive disease of the supraaortic trunks, right carotid-to-left carotid bypass graft has been investigated as a potential alternative to treat patients with arch lesions and deemed unfit for conventional repair. While results have been promising in some series, experts have questioned the feasibility and durability of such types 
of extra-anatomic reconstruction based on the anatomic observation that the distance between the two target vessels is generally not enough to guarantee safe sealing. Points of concern include: the length of the intervention, which includes the carotid-subclavian bypass, the potential risk of stroke, and prosthetic graft infection [reported in less than $4 \%$ of cases (16)]. This type of reconstruction is feasible both for elective cases and in an emergency setting, when conventional open repair would not be tolerated by a fragile patient. Left hemiarch repair using a "hybrid" approach with TEVAR has also been described using a median sternotomy: in this case, a median mini-sternotomy is used to gain access to the origin of the left common carotid artery and to the brachiocephalic trunk in the chest; at that time, a left common carotid-to-brachiocephalic trunk transposition can be performed. Indeed, this approach is more traumatic but guarantees an anatomical reconstruction (thus creating a sort of surgical bovine trunk) with a longer landing zone (17).

\section{Total endovascular options for cerebral revascularization}

As for distal aortic arch lesions, the use of the parallel graft technique has been described to maintain antegrade flow into the supra-aortic vessels. From a technical perspective, even a "triple barrel technique" is feasible and has been reported (18-20). This is a viable solution for emergent cases or for those patients with "no more room" for a conventional repair, either because of clinical or technical aspects. Concerns have been raised around the parallel graft technique regarding technical difficulty and serious clinical consequences (18-20). First, TEVAR with ascending chimneys is not recommended with a proximal aortic neck diameter up to $42 \mathrm{~mm}$ (19). Further, from a technical point of view, ideally the radial force exerted from the aortic endograft should not compromise the chimney graft, while also maintaining an adequate mutual wall apposition. Despite the lack of a dedicated mathematical algorithm, endograft oversizing is suggested to be up to $30 \%$. In addition, the incidence of "gutter-related" type 1a endoleak, which ranges from $0 \%$ to $44 \%$ (18), and the procedurerelated stroke rate is still significant ranging between $4 \%$ and $7.8 \%(20)$.

\section{Single branched endograft}

Currently, there are two devices intended for the total endovascular treatment of arch pathologies: the Gore ${ }^{\circledR}$ TAG $^{\circledR}$ thoracic branched endoprosthesis (W.L. Gore ${ }^{\circledR}$ and ass.; Flagstaff, AZ, USA) and the Valiant MonaLSA ${ }^{\circledR}$,
(Medtronic ${ }^{\circledR}$ Inc.; Minneapolis, MN, USA). The GORE TAG device is built with a pre-cannulated internal retrograde branch, which is either $8 \mathrm{~mm}$ (for "zone 2" lesion) or $12 \mathrm{~mm}$ (for "zone 0" lesion) (Figure $5 \mathrm{~A}$ ). The Valiant MonaLSA device presents the separate side-branch component which is conformed with a proximal flare with an intended $1 \mathrm{~cm}$ overlap to enhance proximal seal. While both the ongoing trials have been designed to evaluate device use for LSA preservation, it is expected to use these new technologies to treat more proximal sealing zones (21).

\section{Operation: hybrid procedure for arch repair}

The patient is positioned in the supine position. This type of intervention should always be performed under general anesthesia, heparinization and neurologic monitoring (transcranial-Doppler, EEG). Surgical access to the common carotid artery is performed with a standard approach bilaterally at the base of the neck. On the left side, an "L-shape" incision is performed to allow easier access to the LSA. The prosthetic bypasses are generally tunneled anteriorly in the suprajugular fossa but a retropharyngeal route has also been described as feasible (16). Prosthetic graft bypasses have been described with a variety of technical variants, including the use of separated grafts to the left common carotid and LSA, or with the use of a preconstituted bifurcated graft, or with a direct bypass from the right common carotid artery to the LSA and an end-toside transposition of the left common carotid artery onto the prosthetic graft. To prevent a type 2 endoleak, the LSA should be embolized by positioning a vascular plug proximal to the origin of the LVA (Figure 5B).

\section{Operation: totally endovascular arch repair}

When using parallel or branched grafts, the technical aspects are no different from those for LSA preservation except that multiple retrograde access from both the common carotid arteries should be used for positioning and releasing all the covered stents parallel to the aortic endograft (Figure 5C). A very important point during these maneuvers is the presence of transient, quick carotid clamping to prevent distal embolization at the moment of the covered stent release. Both the full heparinization and the adjunct of a rapid ventricular pacing may limit thrombus formation and favor the precise deployment of the devices. For the Najuta fenestrated graft, the vital maneuver of inserting and advancing the device is achieved 

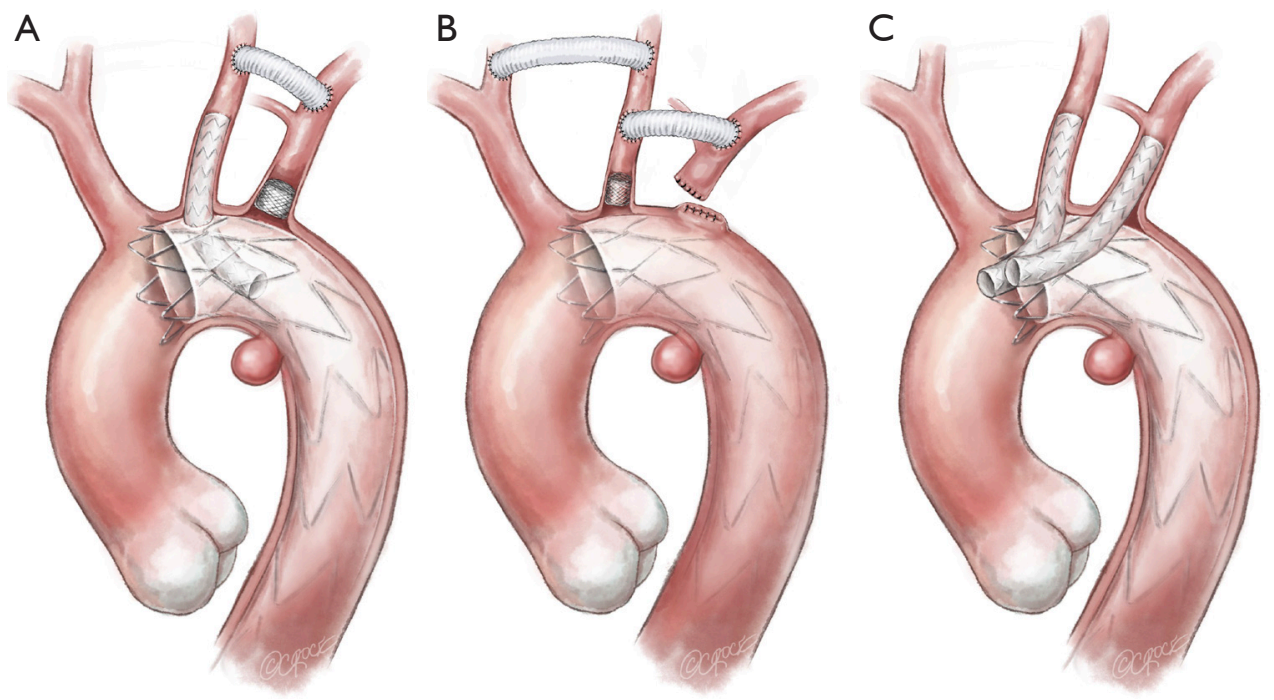

Figure 5 Hybrid and total endovascular techniques for treating PAU which require TEVAR in landing zone 1. (A) Single branched endograft (Gore ${ }^{\circledR}$ TAG $^{\circledR}$ thoracic branched endoprosthesis from W.L. Gore ${ }^{\circledR}$. (B) Endograft deployment in landing zone 1 with surgical carotid-to-carotid bypass and left carotid-LSA bypass grafts plus plug embolization of the LSA. (C) Double parallel technique using a covered stent for the left common carotid artery. The LSA and TEVAR completion is shown. PAU, penetrating aortic ulcer; LSA, left subclavian artery; TEVAR, thoracic endovascular aortic repair.

by the through-and-through access from the right brachial artery. This improves the trackability and pushability of the device introduced through the groin.

\section{TEVAR options for arch PAU with landing zone 0}

\section{Parallel grafts (using covered stent-graft parallel to thoracic endograft)}

Similar to "zone 1" cases, the parallel graft technique is feasible and a potentially reasonable alternative for emergent cases or patients deemed unfit for open repair. In case of "zone 0" lesions, it is possible to use: (I) only one parallel graft for the brachiocephalic trunk in association with a carotid-to-carotid and LSA bypass graft; or (II) two parallel grafts for the brachiocephalic trunk and right common carotid artery, with/without the LSA bypass graft; or finally (III) three parallel grafts to preserve antegrade flow to all the supra-aortic vessels. A common finding after this type of total endovascular approach is a high incidence of "gutter-related" type 1a endoleak that generally has been described to increase with the increased number of chimney grafts (18-20).

\section{Branched stent graft}

Both transverse/proximal aortic arch lesions are the ideal target for branched endografts in patients unfit for open conventional repair. The current available devices, namely the Zenith ${ }^{\circledR}$ arch branched device (Cook Medical; Bloomington, IN; USA), Nexus ${ }^{\circledR}$ (Endospan; Herzlia, Israel), Inoue ${ }^{\circledast}$ stent-graft (PTMC; Kyoto, Japan), and Relay $\mathrm{NBS}^{\circledR}$ Plus (Terumo Aortic; Tokyo, Japan) are actually devices developed for arch repair using a total endovascular approach. Currently they are not available on the market because only very few high-volume centers have been accredited to use them. As occurred for pure ascending TEVAR, arch branched technology is constrained by anatomical landmarks, aortic sizing, and curvatures. While technical success was in the $84-100 \%$ range, mortality rate has been reported up to $20 \%$ and stroke rate up to $40 \%$ due to extensive manipulation in severely diseased aortas (10,21-23). However, a significant reduction over time due to learning curve effects has been reported and the incidence of type 1a endoleak has also been reported as less than that after total debranching $(10,24)$ (Figure $6 A, B)$.

\section{Fenestrated graft}

More recently, custom made endografts have been developed for arch disease. The Najuta endograft is a precurved stent with 64 patterns of stent frame and 21 patterns of large fenestrations to accommodate the orifices of the 

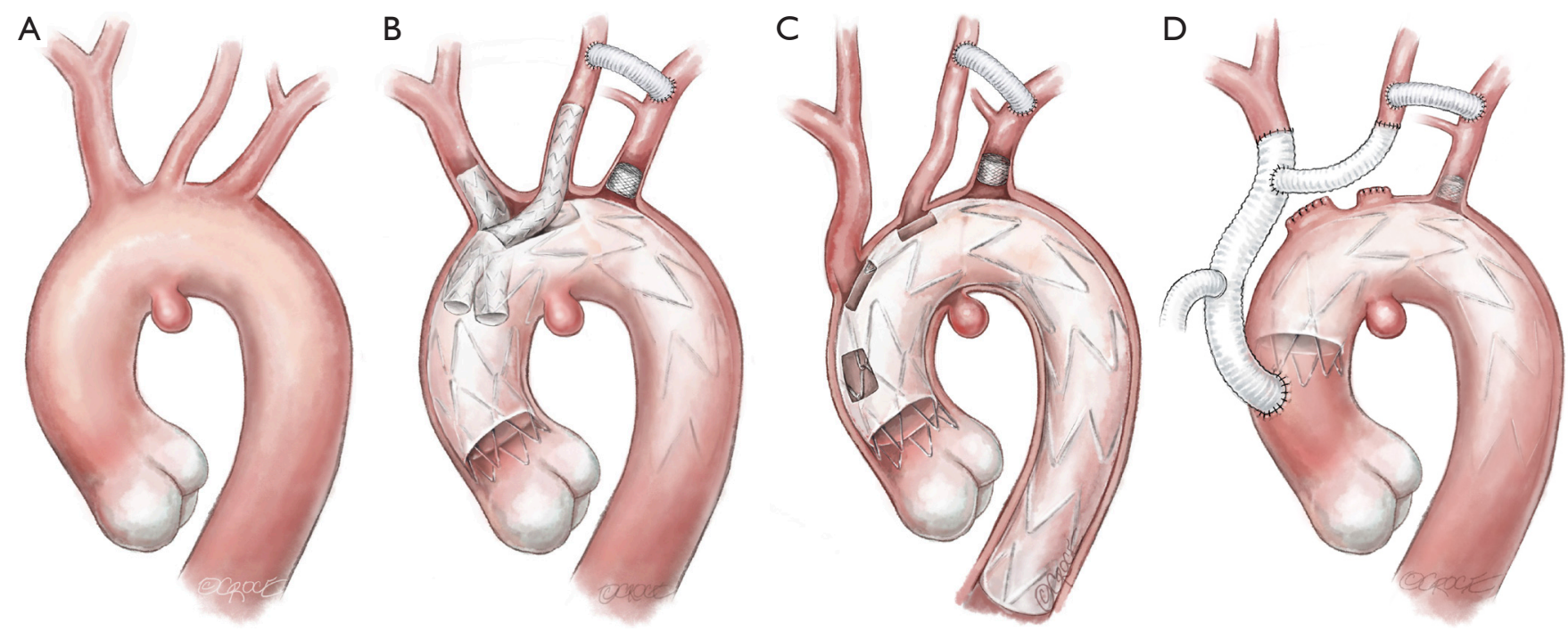

Figure 6 Hybrid and total endovascular techniques for treating PAU which require TEVAR in landing zone 0. (A) Penetrating aortic ulcer (PAU) of the inner curve of the aortic arch which requires proximal TEVAR deployment in Ishimaru zone 0. (B) Double branched Relay NBS $^{\circledR}$ Plus (Terumo Aortic; Tokyo, Japan). (C) Fenestrated endograft Najuta (Kawasumi Inc.; Tokyo, Japan). (D) Type I "hybrid” aortic arch. TEVAR, thoracic endovascular aortic repair.

supra-aortic vessels (Kawasumi Inc.; Tokyo, Japan). The delivery system is available with three different degrees of pre-curvature to facilitate delivery and positioning. The device has been designed to treat mainly saccular aneurysm of the distal aortic arch or lesions developing from the concavity of the arch curvature by placing its large fenestrations across the origins of great vessels with no stent reinforcement. Early mortality and stroke rate seem to be less than other hybrid or endovascular options, but with a higher incidence of type Ia endoleak through fenestrations (32.4\%) (25) (Figure 6C).

\section{Arch debranching}

"Hybrid" aortic arch operations have been classified according to the extent of proximal and distal landing zones, and the need for circulatory support in the form of cardiopulmonary bypass and circulatory arrest. Type I debranching defines an operation which is performed off-pump with aortic side-biting clamping and bypass graft debranching of all supra-aortic vessels (Figure $6 D$ ). Sometimes this type of procedure could be more easily performed on-pump, with circulatory support to better manage more challenging cases such as porcelain aorta or enlarged $(\geq 40 \mathrm{~mm})$ aorta that could be prone to complications such as rupture, bleeding or retrograde dissection. In contrast, type II and III "hybrid" aortic arch repair require circulatory support and/or circulatory arrest with selective antegrade cerebral perfusion to perform conventional graft replacement of the ascending aorta and/ or the transverse arch, respectively. This type of intervention is optimized by using pre-constituted, multibranched grafts with the supra-aortic branches already incorporated in a hemodynamically favorable geometry on the main body of the prosthesis $(17,26,27)$.

\section{Comments}

Open surgical repair is still the gold standard treatment for aortic arch diseases, primarily for aneurysms and dissections, however new devices and techniques for total/ hybrid endovascular repair are already available to treat aortic arch PAUs. These patients are usually older and present with severe co-morbidities, making their surgical profile high-risk or even prohibitive for open repair when compared to those with dissection or aneurysms.

Standardization in such complex cases is difficult; aside from the nature of the aortic lesion, the variability of the anatomy of the arch and its branches can significantly complicate any attempt at using a standardized model. Biomedical industries are continually improving the technology in their products in attempts to provide devices to treat more challenging disease presentations and 
anatomy. Current areas of concern include better stentgraft accommodation and conformability, the correct sizing and location of the fenestrations, and the reduction of existing mismatch between the soft native aorta and the stiffer endografts (23).

In this potentially challenging setting, user experience with different devices is crucial in the decision-making process, however, the learning curve related to the use of new devices should be accounted for, particularly given that its effects are usually associated with sub-optimal outcomes. Additionally, mid- and long-term results are still lacking for all stent-grafts and surgical techniques.

Although these limitations are important, populations are aging more world-wide and surgeons will continue to face increasingly difficult disease presentations and anatomy in coming years. The options for treating them with a minimally-invasive approach, even if associated with minimally-invasive open techniques such as extra-thoracic bypasses, will need to be improved and validated by future experience.

\section{Acknowledgments}

None.

\section{Footnote}

Conflicts of Interest: Dr. Trimarchi is speaker and consultant for Gore (W.L. Gore; Flagstaff, AZ, USA) and Medtronic (Medtronic, Inc.; Minneapolis, MN, USA).

\section{References}

1. Coady MA, Rizzo JA, Hammond GL, et al. Penetrating ulcer of the thoracic aorta: what is it? How do we recognize it? How do we manage it? J Vasc Surg 1998;27:1006-15; discussion 1015-6.

2. Stanson AW, Kazmie FJ, Hollier LH, et al. Penetating atherosclerotic ulcers of the thoracic aorta: natural history and clinicopathologic correlations. Ann Vasc Surg 1986;1:15-23.

3. Patel PJ, Grande W, Hieb RA. Endovascular management of acute aortic syndromes. Semin Intervent Radiol 2011;28:10-23.

4. Nathan DP, Boonn W, Lai E, et al. Presentation, complications, and natural history of penetrating atherosclerotic ulcer disease. J Vasc Surg 2012;55:10-5.

5. Tittle SL, Lynch RJ, Cole PE, et al. Midterm follow-up of penetrating ulcer and intamural hematoma of the aorta. J Thorac Cardiovasc Surg 2002;123:1051-9.

6. Riambau V, Bockler D, Braunkwall J, et al. Editor's choice management of descending aorta disease: clinical practice guidelines of the European Society for Vascular Surgery (ESVS). Eur J Vasc Endovasc Surg 2017;53:4-52.

7. Jánosi RA, Gorla R, Tsagakis K, et al. Thoracic endovascular repair of complicated penetrating aortic ulcer: an 11-year single centre experience. J Endovasc Ther 2016;23:150-9.

8. Oderich GS, Kärkkäinen JM, Reed NR, et al. Penetrating aortic ulcer and Intramural Hematoma. Cardiovasc Intervent Radiol 2019;42:321-34.

9. LeMaire SA, Price M, Parenti J, et al. Early outcomes after aortic arch replacement using the Y-graft technique. Ann Thorac Surg 2011;91:700-7.

10. Maurel B, Mastracci T, Spear R, et al. Branched and fenestrated options to treat aortic arch aneurysms. J Cardiovasc Surg (Torino) 2016;57:686-97.

11. Riambau V. Application of the Bolton Relay Device for Thoracic Endografting In o Near the Aortic Arch. AORTA 2015;3:16-24.

12. Amiot S, Haulon S, Becquemin JP, et al. Fenestrated endovascular grafting: the French multicenter experience. Eur J Vasc Endovasc Surg 2010;39:537-44.

13. Erbel R, Aboyans V, Boileau C, et al. 2014 ESC Guidelines on the diagnosis and treatment of aortic diseases: Document covering acute and chronic aortic diseases of the thoracic and abdominal aorta of the adult. The Task Force for the Diagnosis and Treatment of Aortic Diseases of the European Society of Cardiology (ESC). Eur Heart J 2014;35:2873-926.

14. Yang J, Xiong J, Liu X, et al. Endovascular chimney technique of aortic ach pathologies: a systematic review. Ann Vasc Surg 2012;26:1014-21.

15. van Bogerijen GHW, Williams DM, Eliason JL, et al. Alternative access techniques with thoracic endovascular aortic repair, open iliac conduit versus endoconduit technique. J Vasc Surg 2014;60:1168-76.

16. Gombert A, van Issum L, Barbati ME, et al. ExtraThoracic Supra-aortic Bypass Surgery Is Safe in Thoracic Endovascular Aortic Repair and Arterial Occlusive Disease Treatment. Eur J Vasc Endovasc Surg 2018;55:861-6.

17. Czerny M, Schmidli J, Carrel T, et al. Hybrid aortic arch repair. Ann Cardiothorac Surg 2013;2:372-7.

18. Lindblad B, Bin Jabr A, Holst J, et al. Chimney grafts in aortic stent grafting: hazardous or useful technique? Systematic review of current data. Eur J Vasc Endovasc 
Surg 2015;50:722-31.

19. Shahverdyan R, Mylonas S, Gawenda M, et al. Singlecenter mid-term experience with chimney-graft technique for the preservation of flow to the supra-aortic branches. Vascular 2018;26:175-82.

20. Moulakakis KG, Mylonas SN, Dalainas I, et al. The chimney-graft technique for preserving supra-aortic branches: a review. Ann Cardiothorac Surg 2013;2:339-46.

21. Rana MA, Oderich GS. Current Device designs to incorporate supra-aortic arch trunks. In: Oderich G. Endovascular Aortic Repair. Springe, 2017:501-6.

22. van Bakel TM, de Beaufort HW, Trimarchi S, et al. Status of branched endovascular aortic arch repair. Ann Cardiothorac Surg 2018;7:406-13.

23. Kondov S, Kreibich M, Rylski B, et al. The endovascular repair of the aortic arch using a double branched

Cite this article as: Lomazzi C, Grassi V, Domanin M, De Vincentiis C, Piffaretti G, Trimarchi S. Art of operative techniques: treatment options in arch penetrating aortic ulcer. Ann Cardiothorac Surg 2019;8(4):500-508. doi: 10.21037/ acs.2019.07.06 prosthesis. J Vis Surg 2018;4:132.

24. Spear R, Haulon S, Ohki T, et al. Editor's choice - subsequent results for arch aneurysm repair with inner branched endografts. Eur J Vasc Endovasc Surg 2016;51:380-5.

25. Iwakoshi S, Ichihashi S, Itoh $\mathrm{H}$, et al. Clinical outcomes of thoracic endovascular aneurysm repair using commercially available fenestrated stent graft (Najuta endograft). J Vasc Surg 2015;62:1473-8.

26. Preventza O, Tan CW, Orozco-Sevilla V, et al. Zone zero hybrid arch exclusion versus open total arch replacement. Ann Cardiothorac Surg 2018;7:372-9.

27. Kurimoto Y, Maruyama R, Ujihira K, et al. Thoracic endovascular aortic repair for challenging aortic arch disease using fenestrated stent grafts from zone 0 . Ann Thorac Surg 2015;100:24-32. 